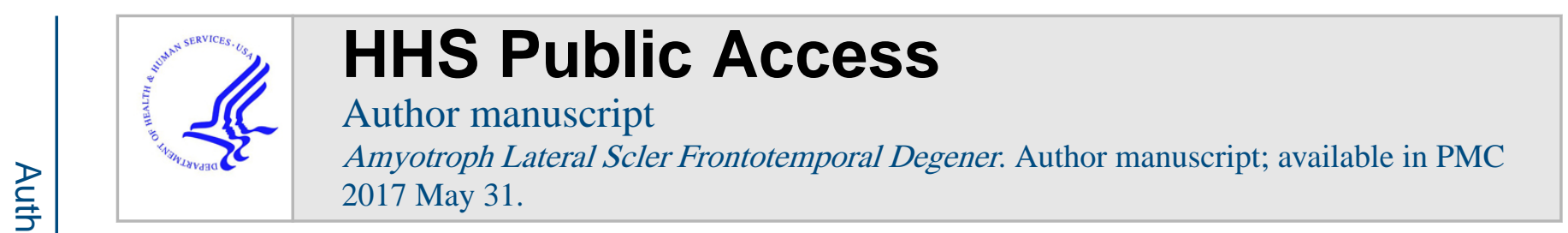

Published in final edited form as:

Amyotroph Lateral Scler Frontotemporal Degener. 2013 May ; 14(4): 267-272. doi:

10.3109/21678421.2012.754043.

\title{
Severe Head Injury and Amyotrophic Lateral Sclerosis
}

\author{
Ms. Tracy L. Peters ${ }^{1}$, Dr. Fang Fang ${ }^{1}$, Mrs. Caroline E. Weibull ${ }^{1}$, Dr. Dale P. Sandler ${ }^{2}$, Dr. \\ Freya Kamel ${ }^{2}$, and Dr. Weimin $\mathrm{Ye}^{1}$ \\ ${ }^{1}$ Department of Medical Epidemiology and Biostatistics, Karolinska Institutet, Stockholm, Sweden \\ ${ }^{2}$ Epidemiology Branch, National Institute of Environmental Health Sciences, Research Triangle \\ Park, North Carolina, USA
}

\begin{abstract}
Objective-To examine whether severe head injury, subtypes of head injury, or repeated head injuries are associated with ALS risk based on Swedish population and health registers.

Methods-We conducted a case-control study, nested within a cohort of 5,764,522 individuals who were born in Sweden during 1901-1970 and followed between 1991 and 2007. The study included 4,004 ALS patients identified from the Swedish Patient Register during follow-up and 20,020 randomly selected controls matched by sex and birth year. We evaluated hospitalization for severe head injury that was recorded in the Inpatient Register before ALS diagnosis. Conditional logistic regression was used to estimate odds ratios (ORs) and 95\% confidence intervals (CIs).

Results-There was an association of ALS risk with severe head injury $\leq$ year before diagnosis (OR: 3.9, 95\% CI: 2.6-6.1). No association was observed for severe head injury $>3$ years before ALS diagnosis, nor was ALS associated with subtypes of head injury or repeated injuries occurring $>3$ years before diagnosis.
\end{abstract}

Conclusions-Our findings from the Swedish registers provide no strong support for an etiological relationship between severe head injury in adulthood and ALS risk.

\section{Keywords}

Amyotrophic Lateral Sclerosis; Head Injury; Risk Factors; Nested Case-Control Study; Motor Neuron Disease

\section{Introduction}

The etiology of ALS likely involves both genetic and environmental factors $(1,2)$. The latter may include metals, smoking, and electrical shock (3-5). Head injury has also been suggested to be a risk factor for ALS. Most studies of this issue have been case-control studies, many of which have collected information on head injury through self-report via

Corresponding Author: Tracy L. Peters (tracy.peters@ki.se) or Weimin Ye (weimin.ye@ki.se), Department of Medical Epidemiology and Biostatistics, Karolinska Institutet, P.O. Box 281, 17177 Stockholm, Sweden.

Declaration of interest:

The views and opinions expressed in this article are those of the authors and should not be construed to represent the views of any of the sponsoring organizations, agencies, or the U.S. Government. All authors report no disclosures. 
mailed questionnaires or interviews (6-11). Other case-control studies have collected information on head injury through review of pre-existing medical records (12-15). Chen et al. (10) reported a significant association for head injury overall in a meta-analysis of eight studies; however, some of the studies in the meta-analysis may have been influenced by recall bias. Armon and Nelson (16) conducted an evidence based review restricted to four studies that evaluated head injury using medical records, and concluded that a single instance of head trauma was unlikely to be a risk factor for ALS. Two studies to date have examined head injury and ALS prospectively. The Oxford Record Linkage Study found that head or other physical injury requiring hospitalization was not a risk factor for ALS overall, while head injury that happened in close proximity to the diagnosis of ALS was associated with a higher risk of ALS (17). The authors of this paper concluded that this apparent higher risk was related to early symptoms prior to formal diagnosis, rather than a primary causative factor. Williams et al. (18) reviewed medical records of head injury prospectively from 1935 to 1974 to evaluate associations with various neurological disorders (including ALS); and there was no evidence of head injury being a risk factor for ALS.

Repeated head injuries may be of special importance for ALS. Chen et al. (10) found repeated head injuries to be associated with an 11-fold risk of ALS. Increased risk of ALS among Italian soccer players was initially proposed to be a result of repeated head injury sustained while "heading" the ball (3). However, an updated study suggested that the excess risk among Italian soccer players might result from a complex interaction between drugs, herbicides and a genetic predisposition for physical endurance (19). More recently, a large population-based case-control study found that ALS was associated with repeated traumatic events, defined as any accidental event causing an injury that required medical care; repeated head injuries in particular were associated with ALS (15). McKee et al. (1) found that some individuals, who were involved in collision sports and experienced repeated head injuries, had CTE where the TDP-43 proteinopathy extended to involve the spinal cord and also had motor neuron degeneration. The authors proposed that CTE might be associated with the development of motor neuron disease but did not consider that they could be seeing two diseases that are unrelated to each other (20-22).

The main goal of this study was to investigate whether severe head injury is a risk factor for ALS in a study based on the Swedish population and health registers, in which information on head injury and ALS was independently and prospectively collected. We specifically aimed to examine the influence of different subtypes of head injury as well as repeated head injuries on ALS risk.

\section{Material and Methods}

\section{Swedish registry data}

The National Patient Register (NPR) was started in 1964/1965 by the National Board of Health and Welfare to collect information on all hospitalizations and has achieved nationwide coverage from 1987 onward [Inpatient Register (IPR)]. In 2001 the NPR also began to collect information on specialist visits [Outpatient Register (OPR)]. Information recorded in the NPR includes the dates of admission, discharge, specialist visits, and up to eight discharge diagnoses. The National Board of Health and Welfare has also been 
responsible for the Causes of Death Register since 1952 (23). Information from this register includes date of death, age of death, underlying cause of death, and contributory causes of death.

The Swedish Population and Housing Census is conducted by Statistics Sweden every five years. The Census is based on a household questionnaire including questions about marital status, household size, housing, employment, occupation, income, and education (23). The Migration Register is part of the National Population Register and records information on immigration and emigration status of registered residents in Sweden; it is updated every few months.

\section{Study cohort and follow-up}

The study cohort was composed of 5,764,522 individuals who were born in Sweden during 1901-1970 and who were included in the 1990 Swedish Population and Housing Census (24). The follow-up started on January $1^{\text {st }}$, 1991, given the nationwide coverage of the IPR in 1987 and the completion of the 1990 Census. We linked the study population to the NPR, the Migration Register, and the Causes of Death Register using the National Registration Number, a unique identifier for all residents in Sweden. Through these linkages we excluded individuals that had died, emigrated out of Sweden or had been diagnosed with ALS before entry to the cohort. The study population was followed from January $1^{\text {st }}, 1991$ until first ALS diagnosis, migration out of Sweden, death, or end of follow-up (December 31 ${ }^{\text {st }}, 2007$ ) whichever came first.

\section{Nested case-control study}

We conducted a case-control study nested within the above-mentioned cohort which was followed, on average, for 14 years, yielding a study base of 83,988,360 person-years.

\section{Cases}

During follow-up, a total of 4,004 individuals were identified with a hospital contact for ALS recorded in the NPR. ALS diagnosis (either as the main diagnosis or a secondary diagnosis at discharge or specialist visit) was identified in the IPR from 1991 to 2007 and in the OPR from 2001 to 2007. ALS diagnoses were recorded according to the International Classification of Diseases, Ninth Revision (ICD-9; 1987-1996, 335C) and Tenth Revision (ICD-10; since 1997, G12.2). Date of the first-ever hospital contact for ALS was defined as the index date for cases.

\section{Controls}

We randomly selected 20,020 controls (five per ALS case) from the study base, using incidence density sampling, i.e. the control had to be alive and in Sweden at the cases' index date. Controls were individually matched with the ALS cases on the basis of age (same birth year) and sex. The index date for controls was defined as the index date of the matched case.

\section{Exposure}

Our exposure of interest was hospitalization for head injury recorded in the IPR (ICD-9. 800,801 and 803 [fracture]; 850 [concussion]; 852 and 853 [hemorrhage]; 851 and 854 
[contusion], and ICD-10: S020, S021, S027, S028, and S029 [fracture]; S060 [concussion]; S061, S062, S063; S064, S065 and S066 [hemorrhage]; S061, S062, S063, S067, S068, S069, S070, and S071 [contusion]). We included both first and secondary diagnoses of head injury occurring before the index date.

\section{Statistical analyses}

Conditional logistic regression modeling was used to estimate odds ratios (ORs) and 95\% confidence intervals (CIs). We first evaluated any severe head injury, followed by subtypes of head injury and repeated head injuries. Repeated head injuries were identified as multiple hospitalizations for distinct head injuries; consecutive transfers among different clinics were counted as one hospitalization. We tested the potential temporal relationship between head injury and ALS by examining the associations between head injuries experienced in different time windows before the index date. Assuming a potential lag between the real onset of ALS and the first hospital contact for ALS, we conducted further analyses examining only head injury experienced $>3$ years before the index date. In all regression models, we adjusted for socioeconomic status and residence regions within Sweden. All statistical analyses were performed using SAS version 9.3 (SAS Institute, North Carolina, USA).

\section{Results}

Among cases, there were more men (56\%) than women (44\%). The largest proportion of cases was in the age groups of 60-69 (28\%) and 70-79 (35\%) years (Table 1). There was a statistically significant association between first severe head injury experienced $\leq$ year before the index date and a higher risk of ALS (OR: 3.9, 95\% CI: 2.6-6.1) (Table 2). There was no association between first severe head injury and ALS at longer lag times before the index date (Table 2). For a first severe head injury experienced $>3$ year before the index date, an OR of 1.2 (95\% CI: 0.9-1.5) was observed.

No association with ALS was seen for any subtype of head injury occurring more than three years before diagnosis, although the estimates were based on small numbers (Table 3). Concussion, which was the most common head injury among both the cases $(\mathrm{n}=51)$ and controls ( $\mathrm{n}=219)$, had an OR of 1.2 (95\% CI: 0.9-1.6) (Table 3).

No association with ALS was found for repeated head injuries occurring more than three years before diagnosis (Table 4). The OR for more than one severe head injury was 0.6 (95\% CI: 0.2-2.0); again the estimate was based on small numbers.

\section{Discussion}

In this prospective study we did not find evidence for an association of ALS with severe head injury overall, with repeated head injuries, or with different subtypes of head injury. A strong association of ALS with head injury experienced $\triangleleft$ year before ALS diagnosis was noted, but is unlikely to be a causal event. Potential reasons for this positive association include first, that incipient disease might make falls and therefore injuries more likely; second, that greater medical surveillance of symptomatic individuals immediately before 
ALS diagnosis might make it more likely that head injury would be recorded; or third, that treatment for a head injury might lead to medical evaluations that would facilitate earlier diagnosis of ALS.

Several reasons potentially explain the inconsistency among studies of severe head injury and the risk of ALS. One factor may be differences in study designs. Some studies showing a significant association between head injury and ALS relied on the recall of head injury (6, 7). Two studies that did not use self-report did not find any association between head injury and ALS $(17,18)$. Our study did not rely on questionnaires or interviews to identify head injury; rather we used independently collected data on previous hospitalizations for head injury. Results of our study are similar to those reported by Turner et al. (17) who examined head injury and ALS using hospital admission records, including day cases (outpatients) and those with a longer stay within the UK National Health Service hospitals. They found an association of ALS with head injury that was restricted to events occurring during the year before ALS diagnosis and concluded that the association was more likely due to incipient ALS precipitating injury rather than to head injury causing ALS (17).

Another potential reason for the difference among studies is that other factors may modify the association between head injury and risk of ALS. Schmidt et al. (25) concluded that head injuries may be a risk factor for ALS but suggested that the strength of association may depend on APOE genotype. Furthermore, Binazzi et al. (11) reported that head injury 11 to 30 years previously was associated with ALS among cases with a bulbar onset. Such findings contribute to inconsistencies in literature but may also provide important insight into the role of head injury in ALS. For example, bulbar onset is also common among soccer players with ALS (19).

Our results showed that in the Swedish population ALS was not associated with repeated severe head injuries recorded in the IPR. These results are inconsistent with those reported by Chen et al. (10) which found that the risk for ALS was 11 times higher among persons who had multiple head injuries occurring within the 10 years before diagnosis of ALS but this finding was based on small numbers and included events occurring shortly before diagnosis. More recently, Pupillo et al (29) reported in a large population-based study that repeated traumatic events occurring at least five years before diagnosis were associated with ALS (OR 2.6 for three or more events). The overall OR for multiple head injuries was 4.8, but results were not given for an analysis of head injury experienced restricted to events five or more years before diagnosis. Both of these studies relied on self-reported information to assess head injury, so recall bias may have been a problem. However, the fact that both studies used structured interviews to collect a complete history of head injury lessens this concern.

Little information is available regarding the role of different subtypes of head injury in ALS. Williams et al. (18) found no association between concussion or skull fracture and ALS (OR: 1.05 , 95\% CI: 0.027, 5.85). Similarly, in the present study we found no association between ALS and any subtype of head injury including concussion, which was the most prevalent head injury among both cases and controls. 
A major strength of our study is the relatively large number of ALS cases and controls, ensuring sufficient statistical power for the main analysis although not for the analyses on subtypes of head injury and repeated head injuries. Furthermore, our study is prospective and thus free of recall bias. However, our study has some limitations. First, because ALS cases were identified from the NPR, our study is limited by a lack of detailed clinical information. For example, our analysis is based on date of first hospital contact for ALS, and the actual date of diagnosis was not available so some injuries could have post-dated ALS diagnosis. Therefore, we used a lag time of three years for some of our analyses to minimize potential reverse causality. Changing this lag time would likely not affect results, because ALS was not related with severe head injury occurring $>1$ year before the index date (data not shown). Second, the quality of ALS diagnosis in NPR has not yet been validated. However, hospital discharge records for ALS diagnosis are of high quality, and the IPR is generally complete and accurate $(26,27)$. Furthermore, validation studies have shown that ICD codes for ALS are a valid diagnostic tool (27-30). In our nested case-control study, we found that among the 4,004 cases of ALS, 2,866 (71.6\%) had at least two hospital admissions with ALS as a diagnosis (main or secondary) in the NPR. Among 692 cases of ALS that were diagnosed between July 2005 and December 2007 when the Drug Prescription Register was available, 459 (66.3\%) had at least one prescription of Riluzole during July 2005-June 2011. The Swedish Prescribed Drug Register was launched on July $1^{\text {st }}, 2005$ and contains prospectively recorded individual data on all dispensed prescribed drugs in Sweden. Third, using hospitalization data from the IPR to identify head injuries limits our study to consideration of head injuries occurring in adulthood and requiring hospitalization. Many head injuries do not require hospitalization, especially ones occurring in children and young adults. Early life exposure to head injuries might potentially be of special importance regarding later development of neurodegeneration. Finally, ALS cases were identified solely from the IPR before 2001, and therefore we may have missed some cases who had sought specialist care but never hospitalized before 2001 .

In summary, we found that there was no association between ALS risk and severe head injury, subtypes of head injury, or repeated head injuries, recorded in the IPR, occurring in adulthood in the Swedish population. The relationship of head injury to ALS has been examined in relatively few high quality studies and merits further study, with particular attention to repeated injuries.

\section{Acknowledgments}

This study was supported by Swedish Research Council (SIMSAM grant No. 80748301 and Grant No. 521-2011-2742), FAS (grant No. 2011-0172), and the Intramural Research Program of The National Institute of Environmental Health Sciences, National Institutes of Health (Z01-ES-049005). FF was supported by postdoctoral fellowships from Hjärnfonden and Svenska Sällskapet för Medicinsk Forskning (SSMF).

\section{References}

1. McKee AC, Gavett BE, Stern RA, Nowinski CJ, Cantu RC, Kowall NW, et al. TDP-43 proteinopathy and motor neuron disease in chronic traumatic encephalopathy. J Neuropathol Exp Neurol. 2010; 69:918-29. [PubMed: 20720505]

2. Mulder DW, Kurland LT, Offord KP, Beard CM. Familial adult motor neuron disease: amyotrophic lateral sclerosis. Neurology. 1986; 36:511-7. [PubMed: 3960325] 
3. Chio A, Benzi G, Dossena M, Mutani R, Mora G. Severely increased risk of amyotrophic lateral sclerosis among Italian professional football players. Brain. 2005; 128:472-6. [PubMed: 15634730]

4. Nelson LM, McGuire V, Longstreth WT Jr, Matkin C. Population-based case-control study of amyotrophic lateral sclerosis in western Washington State. I. Cigarette smoking and alcohol consumption. Am J Epidemiol. 2000; 151:156-63. [PubMed: 10645818]

5. Jafari H, Couratier P, Camu W. Motor neuron disease after electric injury. J Neurol Neurosurg Psychiatry. 2001; 71:265-7. [PubMed: 11459909]

6. Kondo K, Tsubaki T. Case-control studies of motor neuron disease: association with mechanical injuries. Arch Neurol. 1981; 38:220-6. [PubMed: 7011280]

7. Deapen DM, Henderson BE. A case-control study of amyotrophic lateral sclerosis. Am J of Epidemiol. 1986; 123:790-9. [PubMed: 3962963]

8. Gallagher JP, Sanders M. Trauma and amyotrophic lateral sclerosis: a report of 78 patients. Acta Neurol Scand. 1987; 75:145-50. [PubMed: 3577678]

9. Gresham LS, Molgaard CA, Golbeck AL, Smith R. Amyotrophic lateral sclerosis and history of skeletal fracture: a case-control study. Neurology. 1987; 37:717-9. [PubMed: 3561788]

10. Chen H, Richard M, Sandler DP, Umbach DM, Kamel F. Head injury and amyotrophic lateral sclerosis. Am J Epidemiol. 2007; 166:810-6. [PubMed: 17641152]

11. Binazzi A, Belli S, Uccelli R, Desiato MT, Talamanca IF, Antonini G, et al. An exploratory casecontrol study on spinal and bulbar forms of amyotrophic lateral sclerosis in the province of Rome. Amyotroph Lateral Scler. 2009; 10:361-9. [PubMed: 19922125]

12. Kurtzke JF, Beebe GW. Epidemiology of amyotrophic lateral sclerosis: 1. A case-control comparison based on ALS deaths. Neurology. 1980; 30:453-62. [PubMed: 7189251]

13. Granieri E, Carreras M, Tola R, Paolino E, Tralli G, Eleopra R, et al. Motor neuron disease in the province of Ferrara, Italy, in 1964-1982. Neurology. 1988; 38:1604-8. [PubMed: 3419606]

14. Chio A, Meineri P, Tribolo A, Schiffer D. Risk factors in motor neuron disease: a case-control study. Neuroepidemiology. 1991; 10:174-84. [PubMed: 1745327]

15. Pupillo E, Messina P, Logroscino G, Zoccolella S, Chio A, Calvo A, et al. Trauma and amyotrophic lateral sclerosis: a case-control study from a population-based registry. Eur J Neurol. 2012

16. Armon C, Nelson LM. Is head trauma a risk factor for amyotrophic lateral sclerosis? An evidence based review. Amyotrophic Lateral Scler. 2012; 13:351-6.

17. Turner MR, Abisgold J, Yeates DG, Talbot K, Goldacre MJ. Head and other physical trauma requiring hospitalisation is not a significant risk factor in the development of ALS. J Neurol Sci. 2010; 288:45-8. [PubMed: 19878957]

18. Williams DB, Annegers JF, Kokmen E, O’Brien PC, Kurland LT. Brain injury and neurologic sequelae: a cohort study of dementia, parkinsonism, and amyotrophic lateral sclerosis. Neurology. 1991; 41:1554-7. [PubMed: 1922795]

19. Chio A, Calvo A, Dossena M, Ghiglione P, Mutani R, Mora G. ALS in Italian professional soccer players: the risk is still present and could be soccer-specific. Amyotrophic Lateral Scler. 2009; 10:205-9.

20. Appel SH, Cwik VA, Day JW. Trauma, TDP-43, and amyotrophic lateral sclerosis. Muscle \& Nerve. 2010; 42:851-2. [PubMed: 21104858]

21. Bedlack RS, Genge A, Amato AA, Shaibani A, Jackson CE, Kissel JT, et al. Correspondence regarding: TDP-43 proteinopathy and motor neuron disease in chronic traumatic encephalopathy. J Neuropathol Exp Neurol. 2010; 69:918-29. [PubMed: 20720505] J Neuropathol Exp Neurol. 2011; 70:96-7. author reply 8-100. [PubMed: 21173608]

22. Armon C, Miller RG. Correspondence regarding: TDP-43 proteinopathy and motor neuron disease in chronic traumatic encephalopathy. J Neuropathol Exp Neurol. 2010; 69:918-29. [PubMed: 20720505] J Neuropathol Exp Neurol. 2011; 70:97-8. author reply 8-100. [PubMed: 21173609]

23. Lagerlund M, Bellocco R, Karlsson P, Tejler G, Lambe M. Socio-economic factors and breast cancer survival--a population-based cohort study (Sweden). Cancer Causes \& Control. 2005; 16:419-30. [PubMed: 15953984] 
24. Fang F, Chen H, Wirdefeldt K, Ronnevi LO, Al-Chalabi A, Peters TL, et al. Infection of the central nervous system, sepsis and amyotrophic lateral sclerosis. PloS one. 2011; 6:e29749. [PubMed: 22216353]

25. Schmidt S, Kwee LC, Allen KD, Oddone EZ. Association of ALS with head injury, cigarette smoking and APOE genotypes. Journal Neurol Sci. 2010; 291:22-9. [PubMed: 20129626]

26. Fang F, Valdimarsdottir U, Bellocco R, Ronnevi LO, Sparen P, Fall K, et al. Amyotrophic lateral sclerosis in Sweden, 1991-2005. Arch Neurol. 2009; 66:515-9. [PubMed: 19364937]

27. Kurian KM, Forbes RB, Colville S, Swingler RJ. Cause of death and clinical grading criteria in a cohort of amyotrophic lateral sclerosis cases undergoing autopsy from the Scottish Motor Neurone Disease Register. J Neurol Neurosurg Psychiatry. 2009; 80:84-7. [PubMed: 19091712]

28. St Germaine-Smith C, Metcalfe A, Pringsheim T, Roberts JI, Beck CA, Hemmelgarn BR, et al. Recommendations for optimal ICD codes to study neurologic conditions: A systematic review. Neurology. 2012 Epub 2012/08/24.

29. Beghi E, Logroscino G, Micheli A, Millul A, Perini M, Riva R, et al. Validity of hospital discharge diagnoses for the assessment of the prevalence and incidence of amyotrophic lateral sclerosis. Amyotrophic Lateral Scler. 2001; 2:99-104.

30. Pisa FE, Verriello L, Deroma L, Drigo D, Bergonzi P, Gigli GL, et al. The accuracy of discharge diagnosis coding for Amyotrophic Lateral Sclerosis in a large teaching hospital. Eur J Epidemiol. 2009; 24:635-40. [PubMed: 19657715]

31. Wettermark B, Hammar N, Fored CM, Leimanis A, Otterblad Olausson P, Bergman U, et al. The new Swedish Prescribed Drug Register--opportunities for pharmacoepidemiological research and experience from the first six months. Pharmacoepidemiol Drug Saf. 2007; 16:726-35. [PubMed: 16897791] 
Peters et al.
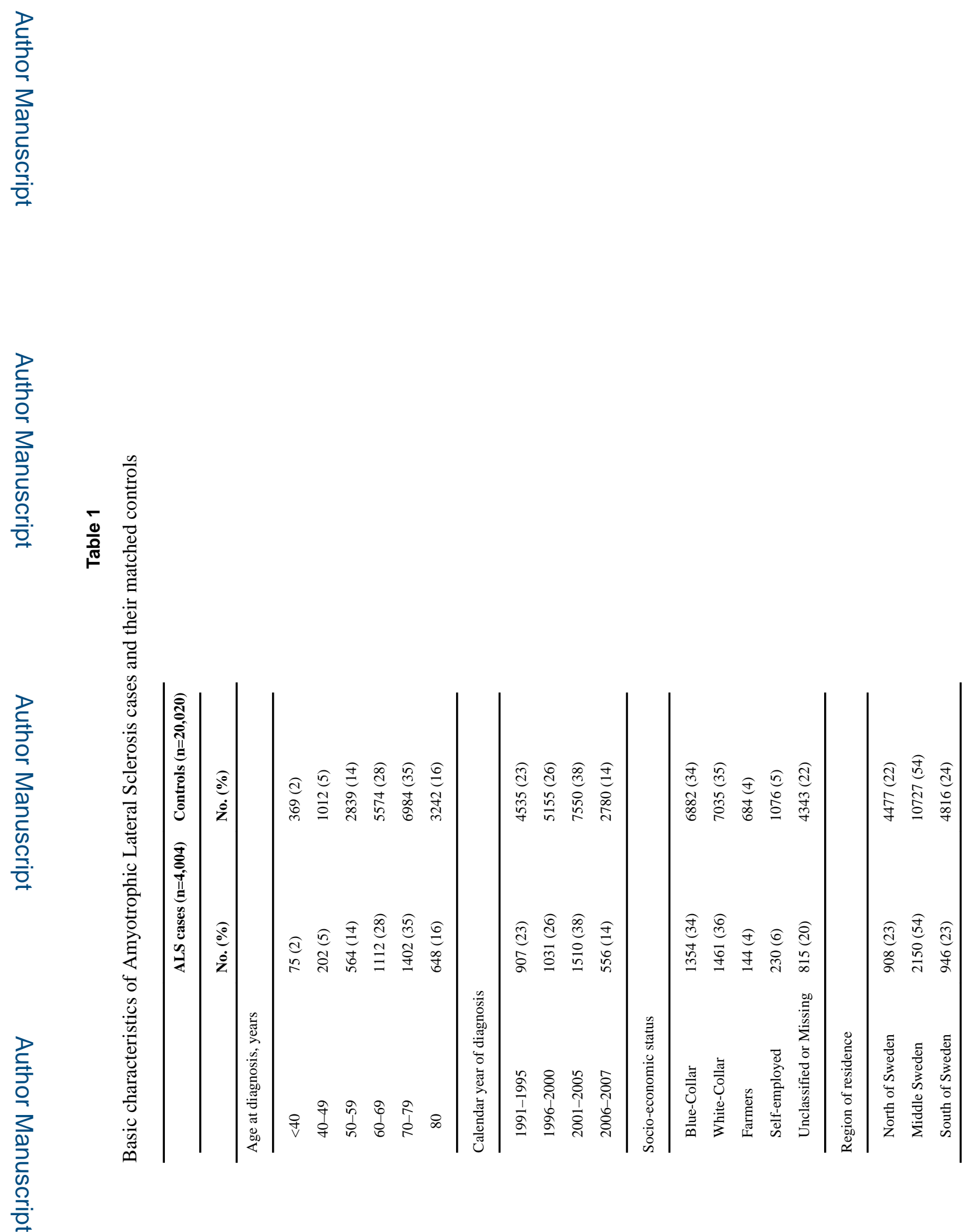

Amyotroph Lateral Scler Frontotemporal Degener. Author manuscript; available in PMC 2017 May 31. 

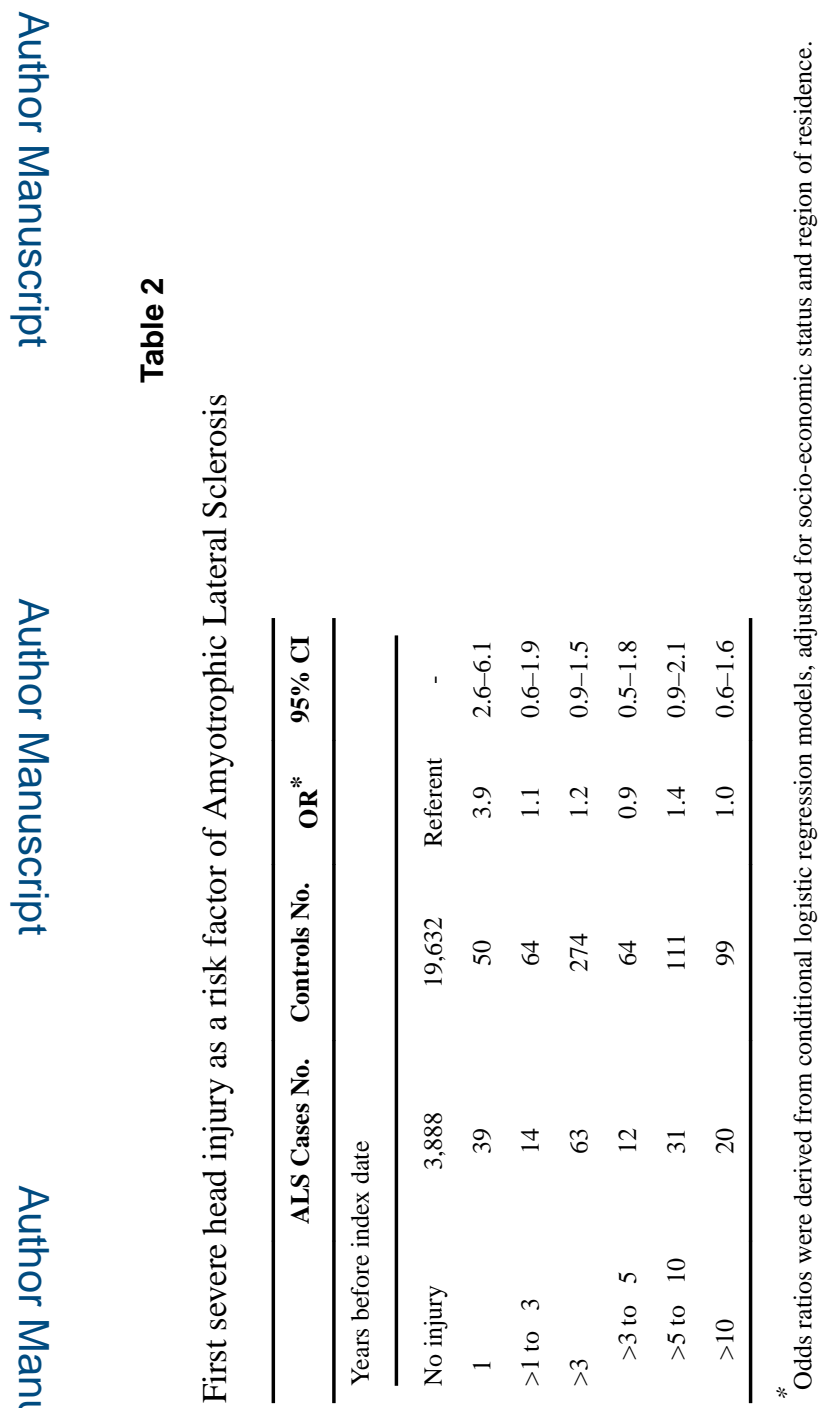


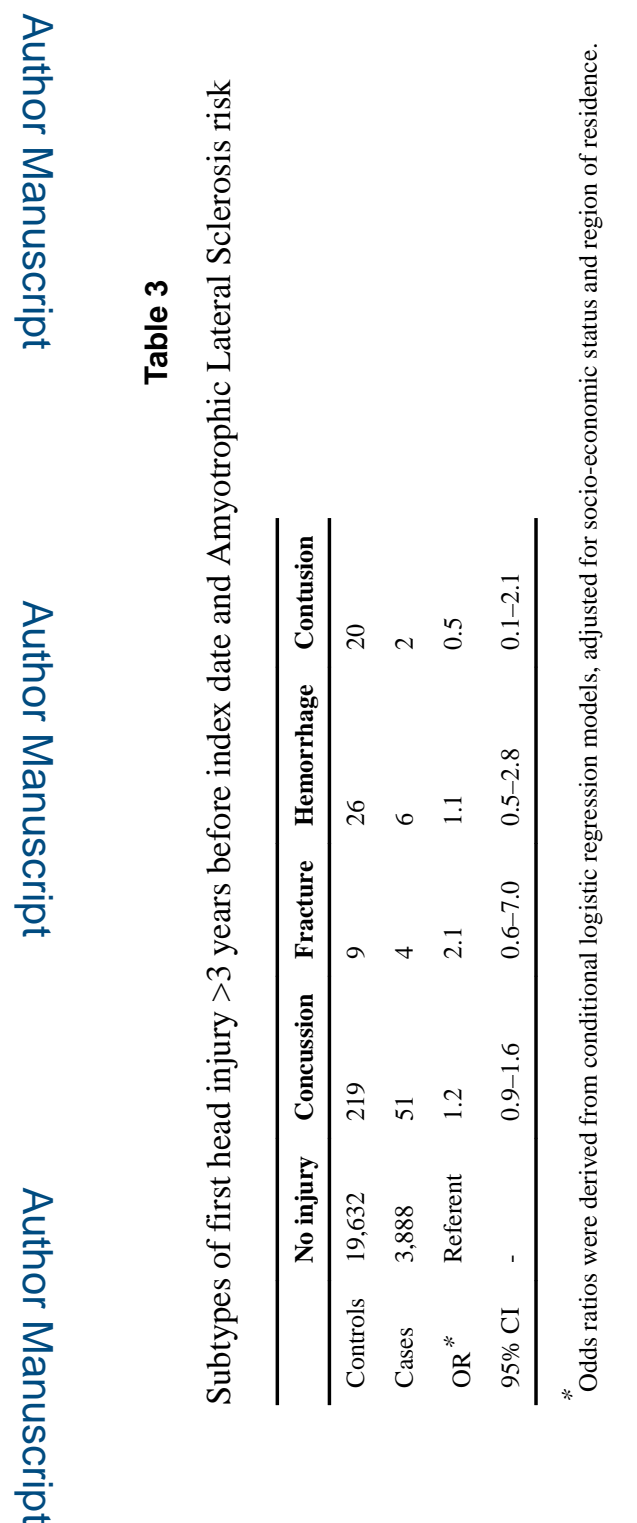



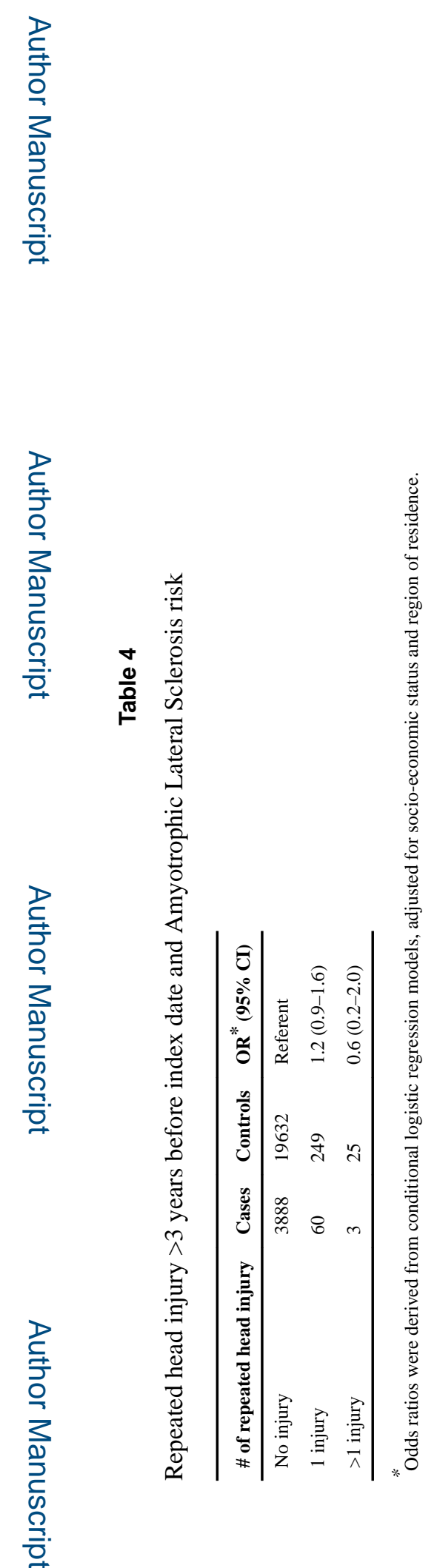

Amyotroph Lateral Scler Frontotemporal Degener. Author manuscript; available in PMC 2017 May 31. 